\title{
Measuring Experiential Avoidance: Reliability and Validity of the Dutch 9-item Acceptance and Action Questionnaire (AAQ)
}

\author{
Paul A. Boelen - Albert Reijntjes
}

Published online: 23 February 2008

(C) The Author(s) 2008

\begin{abstract}
Three studies evaluated psychometric properties of the Dutch version of the 9-item Acceptance and Action Questionnaire (AAQ) - a self-report measure designed to assess experiential avoidance as conceptualized in Acceptance and Commitment Therapy (ACT). Study 1, among bereaved adults, showed that a one-factor model, with AAQ-items constituting a single dimension of experiential avoidance, fitted the data well. The internal consistency and temporal stability of the AAQ were satisfactory. In Study 2, among undergraduate students, and Study 3, among 60 outpatients, higher AAQ scores were found to be significantly associated with psychopathology, maladaptive coping strategies, and neuroticism, attesting to the validity of the measure. In support of its incremental validity, Study 3 showed that the AAQ remained significantly associated with depression and anxiety after controlling for neuroticism and thought suppression. The current studies complement prior research supporting the psychometric properties of the AAQ and the ACT conceptualization of experiential avoidance.
\end{abstract}

Keywords Acceptance and action questionnaire .

Experiential avoidance .

Acceptance and commitment therapy .

Psychometric properties

P. A. Boelen $(\square)$

Department of Clinical and Health Psychology,

Utrecht University,

P.O. Box 80140, 3508 TC Utrecht, The Netherlands

e-mail: P.A.Boelen@uu.nl

\section{A. Reijntjes}

Department of Psychosocial Development in Context,

Utrecht University,

Utrecht, The Netherlands

\section{Introduction}

Experiential avoidance refers to attempts to alter the frequency, duration, or form of negatively evaluated private events such as thoughts, feelings, memories, and the contexts that engender them (e.g., Hayes et al. 1999, 1996). The unwillingness to remain in contact with particular negative internal-subjective experiences falls on the same continuum as psychological acceptance, with higher levels of experiential acceptance reflecting more willingness to experience and endure unwanted private events without judgment and defense (Hayes et al. 1996). As Hayes noted, experiential acceptance "involves experiencing events fully and without defense...and involves making contact with the automatic or direct stimulus functions of events, without acting on the basis solely of their derived verbal functions" (Hayes 1994, p. 30).

Experiential avoidance is not pathogenic by definition. However, chronically or excessively attempting to avoid negative subjective experiences is considered a core psychological vulnerability underlying the onset and/or maintenance of a variety of unfavorable psychosocial and health-related outcomes, ranging from substance abuse to suicide (e.g., Blackledge and Hayes 2001; Hayes et al. 1999). For instance, avoidance behavior in anxiety disorders is generally considered to maintain and exacerbate anxiety-related problems via the process of negative reinforcement, thereby generating a vicious self-perpetuating cycle (Barlow 2002).

Ironically, the generalized tendency to respond to one's negative thoughts and unwanted emotional states in an avoidant fashion in an effort not to have them, often yields increased sympathetic activation, more intense negative emotions, and renders it more likely that unwanted emotional responses will occur again and more severely in 
the future (Gross and Levenson 1997; Hayes et al. 1996). Moreover, the habitual use of experiential avoidance may produce the situation wherein the solutions aimed at controlling or minimizing emotional responding become problems themselves (e.g., not being able to travel in agoraphobia). Parallel to the accumulation of evidence suggesting that the association between stressors and adjustment may occur because of experiential avoidance (i.e. experimental avoidance is a mediator in predictions of distress), a variety of behavior therapy approaches that have incorporated interventions to decrease avoidance patterns have been developed. These include, among others, Dialectical Behavior Therapy (Linehan 1993), MindfulnessBased Cognitive Therapy (Segal et al. 2001), and Acceptance and Commitment Therapy (ACT; Hayes et al. 1999).

In light of the growing recognition that experiential avoidance is important in psychological (mal)functioning, the Acceptance and Action Questionnaire (AAQ) was developed, a self report measure tapping various aspects of acceptance/willingness vs. experiential avoidance. Several versions of the AAQ that contain different numbers of items have emerged. For instance, a 16-item version has been developed and examined by Bond and Bunce (2003), whereas Hayes and colleagues have developed and examined a shorter 9-item version (Hayes et al. 2004). Although it should be noted that the AAQ-II will become available in the near future, at present this latter shorter version is most commonly used in research on ACT (Hayes et al. 2006). The current study therefore focuses on the psychometric properties of this 9-item version.

Across different versions, the development of the AAQ was guided by key assumptions of ACT and its underlying theory of language and cognition, called Relational Frame Theory (RFT; Hayes et al. 2001). According to RFT, the core of human language and cognition is the learned and contextually controlled ability to arbitrarily relate events mutually and in combination, and to change the functions of specific events based on their relations to others. Importantly from a clinical point of view, the nature of human language may markedly increase the range of potential aversive events, because in most contexts symbolic behavior allows for the categorization of private events and people's contact with them. For example, because negatively evaluated subjective feeling states such as anxiety can be recalled or predicted via language (Hayes et al. 2004), humans can start actively avoiding or suppressing negatively evaluated private events such as anxious thoughts.

In a recent series of studies, using different samples including over 2,400 people, Hayes et al. (2004) showed that the 9 items of the AAQ constitute a single dimension of experiential avoidance, and that higher scores on the AAQ are positively linked with several indices of psychopathology and decreased quality of life. Other studies provided additional support for the ACT/RFT conception of experiential avoidance, and the AAQ as a valid tool to tap the phenomenon (for a review see Hayes et al. 2006). Moreover, a study examining the Spanish adaptation of the AAQ has provided preliminary evidence in favour of the reliability and concurrent validity of the measure in a different Western culture (Baracca Mairal 2004).

Notwithstanding the importance of research conducted with the AAQ to date, several issues warrant further study. First, although many studies used the AAQ to assess experiential avoidance (Hayes et al. 2006), few studies were specifically designed to evaluate its psychometric properties. Second, no studies have yet examined versions of the AAQ other than Spanish (Baracca Mairal 2004) or English (Hayes et al. 2004) ones. In light of the rapid expansion of research on ACT/RFT, it is important to further establish the psychometric properties of the AAQ and the cross-cultural generalizability of findings obtained thus far. The overarching aim of the studies presented in this paper was to provide information on the psychometric properties of the Dutch version of the AAQ. In so doing, three independent samples were examined that varied in age and clinical status.

In Study 1, a sample of bereaved adults was used to investigate the factor-structure, internal consistency, and short term temporal stability of the AAQ, as well as its associations with measures of psychopathology. Based on the findings of Hayes et al. (2004), it was expected that a unitary model, with items of the AAQ forming a single dimension of experiential avoidance, would fit the data well and would display adequate internal consistency and test-retest reliability. With regard to concurrent validity, it was predicted that higher scores on the AAQ would be positively associated with symptoms of depression, anxiety, and complicated grief.

In Study 2, a sample of undergraduate students was used to further examine concurrent associations between scores on the AAQ and measures indexing several aspects of psychological functioning. Specifically, participants completed questionnaires tapping depression and anxiety, the frequency and perceived stressfulness of daily hassles, coping style, and dimensions of personality as distinguished in the five-factor model of personality (i.e., neuroticism, extraversion, agreeableness, openness, conscientiousness; McCrae and Costa 1999). It was again predicted that higher scores on the AAQ would be positively associated with depression and anxiety. With respect to the construct validity, it was predicted that scores on the AAQ would be more strongly related to the perceived stressfulness of daily hassles than their actual frequency. This expectation was based on the fact that experiential avoidance is conceptualized as a process that is more strongly associated with the subjective experience of life's adversity than with the actual occurrence of adversity per se (cf. Hayes et al. 2006). Moreover, higher scores on 
the AAQ were expected to be inversely associated with adaptive coping strategies (e.g., active coping) and positively associated with maladaptive coping strategies (e.g., venting of emotions, cf. Carver et al. 1989). Finally, based on previous work (Bond and Bunce 2003; Kashdan et al. 2006), it was expected that experiential avoidance would be positively associated with neuroticism, a widely recognized unhealthy personality dimension. In addition, experiential avoidance was expected to be negatively associated with extraversion, agreeableness, and conscientiousness-all representing relatively healthy features of personality (cf. Costa and McCrae 1992; Durrett and Trull 2005) - and positively associated with openness, a dimension that has been found to be associated with psychological malfunctioning (Trull and Sher 1994).

In Study 3, psychometric properties of the AAQ were further investigated in a clinical sample. Participants completed measures of depression, anxiety, thought suppression, neuroticism, and coping. Experiential avoidance is conceptualized as a process encompassing negative evaluations of private experiences, as well as deliberate attempts to minimize these experiences (Hayes et al. 2006). Therefore, with respect to the construct validity, it was predicted that the AAQ would be significantly correlated with neuroticism, representing the broader tendency to think and feel negatively, as well as with thought suppression, representing one specific manifestation of experiential avoidance. However, experiential avoidance is considered a phenomenon that is associated with, but more inclusive than neuroticism and thought suppression. Hence, it was expected that the AAQ would continue to predict variance in depression and anxiety, when controlling for the shared variance with both constructs (cf. Hayes et al. 2004). Finally, as in Study 2, it was predicted that higher scores on the AAQ would be positively associated with psychopathology and with maladaptive coping strategies and inversely related with adaptive coping strategies.

The selection of the measures employed in the current studies was based on several considerations. First, because experiential avoidance is assumed to be implicated in a wide range of clinical problems and disorders, it was considered important to select measures tapping different relevant domains of psychological functioning. Second, it was deemed important to select measures that were short, easily administered, and well-validated in the Netherlands.

\section{Study 1}

Method

Participants and Procedure Data were available from 404 bereaved adults who were originally recruited for a longitudinal research program on coping with loss. Primary goals of this ongoing program were to examine the predictive effects of various types of coping behaviors on the development of complicated grief symptoms over time. All participants were recruited through an advertisement on an Internet-site that briefly explained aims of the program and invited mourners to participate by filling in questionnaires. Individuals were invited to express their willingness to participate by sending an e-mail to the first author. A digital version of the questionnaire packet was sent to those who did so. Participants could also obtain a paper version of the packet, if so wished. In total, 568 packets were sent out, 404 of which $(71 \%)$ were returned. Data of two respondents below 18 years of age were not included. Informed consent was obtained from all participants. This study was approved by the Institutional Review Board of the Faculty of Social Sciences of Utrecht University, together with the other two studies presented in this paper. The final sample of this study encompassed 402 respondents. The mean age of the participants was $41.8(\mathrm{SD}=12.0)$ years. Most (89\%) were women. On average, participants had had 16.3 $(\mathrm{SD}=3.1)$ years of education. Participants had suffered the loss of a partner $(30.6 \%)$, child $(15.9 \%)$, sibling $(11.7 \%)$, parent $(32.6 \%)$, or other relative $(9.2 \%)$, on average 4 years ago ( $\mathrm{SD}=90.5$ months). In almost half of all cases $(46.8 \%)$, losses were due to illnesses.

The temporal stability of the AAQ was examined with a sub-sample of 30 participants who completed the measure twice. The mean retest interval was of $22.6(\mathrm{SD}=7.4)$ days. All but one were women. On average, participants were 39.0 years of age $(\mathrm{SD}=11.2)$ and had had 15.9 years of education $(\mathrm{SD}=3.4)$.

Measures Participants completed several questionnaires for the research program on coping with loss. The instruments that were used in the current study are presented below.

The 9-item Acceptance and Action Questionnaire (AAQ) was administered. As noted, the AAQ is a self-report measure of experiential avoidance developed by Hayes et al. (2004). Items are shown in Table 1. Items were designed to tap different aspects of experiential avoidance, namely the ability to act effectively when experiencing negatively evaluated private events (items 1 and 3), negative evaluations of private events (items 5 and 8) and painful experiences (item 9), the ability to distance oneself from the literal content of negative evaluations (item 6), the use of worry or daydreaming to regulate behavior (items 2 and 4), and negative comparisons as to how others handle their lives (item 7). Respondents rate the degree to which items apply to them on 7-point scales, ranging from 1 ("never true") to 7 ("always true").

Two studies explicitly focused on the psychometric properties of the 9-item AAQ (Baracca Mairal 2004; Hayes 
Table 1 Items of the acceptance and action questionnaire and factor-loadings and test-retest correlations in Study 1

\begin{tabular}{|c|c|c|}
\hline & $\begin{array}{l}\text { Factor-loadings } \\
\text { in unitary model } \\
(N=402)\end{array}$ & $\begin{array}{l}\text { Test-retest } \\
\text { correlations } \\
(n=30)\end{array}$ \\
\hline $1 \mathrm{I}$ am able to take action on a problem even if I am uncertain what is the right thing to do & -0.53 & $0.66^{* * *}$ \\
\hline 2 I often catch myself daydreaming about things I've done and what I would do differently next time & 0.43 & $0.57 * *$ \\
\hline 3 When I feel depressed or anxious, I am unable to take care of my responsibilities & 0.58 & $0.64 * * *$ \\
\hline 4 I rarely worry about getting my anxieties, worries, and feelings under control & -0.55 & $0.37 *$ \\
\hline 5 I'm not afraid of my feelings & -0.64 & $0.50 * *$ \\
\hline 6 When I evaluate something negatively, I usually recognize that this is just a reaction, not an objective fact & -0.05 & 0.15 \\
\hline 7 When I compare myself to other people, it seems that most of them are handling their lives better than I do & 0.75 & $0.66^{* * *}$ \\
\hline 8 Anxiety is bad & 0.27 & $0.56^{* *}$ \\
\hline 9 If I could magically remove all the painful experiences I've had in my life, I would do so & 0.55 & $0.68 * * *$ \\
\hline
\end{tabular}

Ratings on items $1,4,5$, and 6 are reversed for scoring purposes.

${ }^{*} p<0.05 ;{ }^{* *} p<0.01 ; * * * p<0.001$

et al. 2004). Using data of 460 clients from a university counseling center, Hayes et al. found that a one-factor model fitted the data well and had satisfactory internal consistency $(\alpha=0.70)$. The validity was evaluated using ten different samples. It was found that the AAQ was significantly associated with measures tapping specific, narrowly defined forms of coping (e.g., thought suppression, dissociation, post-traumatic avoidance). Yet, correlations were all below 0.40 , which was interpreted as indicating that the AAQ taps a construct that goes beyond more specific forms of coping. In support of its concurrent validity, the AAQ correlated significantly with measures of depression, anxiety, and other indices of psychological malfunctioning. In support of its incremental validity, the AAQ was linked with several measures of psychopathology, after controlling for selfdeceptive positivity and thought suppression.

Baracca Mairal (2004) recently examined the Spanish version of the AAQ among 48 clinical and 66 non-clinical participants. Results showed that the internal consistency $(\alpha=0.74)$ and 5 to 6 weeks temporal stability $(r=0.71)$ of this version were adequate. In support of its concurrent validity, the AAQ was found to be associated with depressive symptoms, anxiety, and borderline personality traits.

To construct a Dutch version of the instrument, the first author and another psychologist, independent of each other, translated the English version of the AAQ into Dutch and translated each other's version back into English. Discrepancies between both Dutch versions were then discussed and cleared to make sure that the Dutch translation resembled the original formulation as closely as possible. After reversing scores of the items representing acceptance and emotional flexibility (items 1, 4, 5, and 6), item scores are summed to form a composite score of experiential avoidance. Items can be keyed such that higher scores reflect more acceptance and flexibility or, alternatively, such that higher scores reflect more experiential avoidance.
Following Hayes et al. (2004), this latter scoring method was used in the current studies.

Self-reported depression and complicated grief symptoms were used as indices of psychopathology. Depression was assessed with the 16-item scale from the Symptom Checklist (SCL-90; Derogatis 1983). Respondents rate how often they experienced symptoms in the preceding week, on 5-point scales ranging from 1 (never) to 5 (always). Items are summed to yield a depression severity score. The Dutch version of the SCL-90 has demonstrated adequate psychometric properties (Arrindell and Ettema 1986). In the current sample, the Depression scale yielded an $\alpha$ of 0.94 .

Complicated grief was assessed with the 29-item Dutch version of the Inventory of Complicated Grief-revised (ICG-r; original version by Prigerson and Jacobs 2001). Respondents rate the occurrence of complicated grief symptoms in the preceding month on 5-point scales ranging from 1 (never) to 5 (always). Items are summed to form a complicated grief severity score. Psychometric properties of the Dutch ICG-r are adequate (Boelen et al. 2003). Cronbach's $\alpha$ in the present sample was 0.96 .

\section{Results}

Factor Structure CFA was conducted using Amos 5.0 (Arbuckle 2003). To evaluate goodness of fit, several indices were used, including (a) the ratio of $\chi^{2}$ to degrees of freedom $\left(\chi^{2} / d f\right)$ that approaches zero as the fit of the model improves, (b) the Comparative Fit Index (CFI), (c) the Normed Fit Index (NFI), and (d) the Tucker Lewis Index (TLI) that is also known as the non-normed fit index (NNFI) of which values of $>0.90$ indicate acceptable model fit, and (e) the Root Mean Square Error of Approximation (RMSEA) of which values $<0.08$ indicate acceptable fit.

The 9-item unitary model showed a marginally acceptable fit to the data. Fit indices were as follows: $\chi^{2} / d f=3.24, \mathrm{CFI}=$ 
$0.91, \mathrm{NFI}=0.87, \mathrm{TLI} / \mathrm{NNFI}=0.88$, and $\mathrm{RMSEA}=0.08$. Modification-indices indicated that the error-terms of items 1 and 3 were correlated. As both items reflect the inability to take needed action in the face of negative private events, it was assumed that correlations stemmed from similarity in content. Accordingly, the fit of an adjusted model in which these error-terms were allowed to correlate was tested. This model was a significant improvement of the first model $\left(\chi^{2}\right.$ difference $=15.63, \Delta d f=1, p<0.001)$, and demonstrated good fit-estimates $\left(\chi^{2} / d f=2.76, \mathrm{CFI}=0.93, \mathrm{NFI}=0.90, \mathrm{TLI} /\right.$ $\mathrm{NNFI}=0.90$, and RMSEA=0.07). ${ }^{1}$ Item factor-loadings of this second model are shown in Table 1. Item-factor loadings all differed significantly from 0 ( $Z$-scores $>4.53, p$ 's $<0.001)$, except the factor-loading of item $6(Z=0.95, p=0.34)$. Notwithstanding the poor loading of this item, findings were consistent with those of Hayes et al. (2004) in showing that different aspects of experiential avoidance can be conceptualized as a unidimensional concept. The overall experiential avoidance score was calculated by summing the AAQ item scores.

Descriptives The AAQ total score in the current sample was slightly positively skewed (Skewness $=0.29, \mathrm{SE}=0.12$, ratio 2.41; Kurtosis $=-0.12, \mathrm{SE}=0.24)$. The mean AAQ score of participants was $30.5(\mathrm{SD}=7.8)$. Scores of men $(M=29.6$, $\mathrm{SD}=7.5)$ did not differ significantly from those of women $(M=30.6, \quad \mathrm{SD}=7.9 ; t(400)=0.84, p=0.40)$. Mean AAQ scores of men and women in the present sample were compared with the weighted mean AAQ scores of men $(M=$ 34.6, $\mathrm{SD}=7.3)$ and women $(M=37.3, \mathrm{SD}=8.0)$ from the three clinical samples included in the study of Hayes et al. (2004; samples 1, 2, and 5). In addition, scores in the present sample were compared with weighted mean AAQ scores of men from two undergraduate samples (samples 3 and $10 ; M=33.2, \mathrm{SD}=6.5$ ) and women from three undergraduate samples (samples 3, 4, and 10; $M=33.4$, $\mathrm{SD}=6.9$ ) that were included in the research of Hayes et al. Men in the present sample scored lower than men in the clinical samples $(t(43)=-4.45, p<0.001)$ and men in the nonclinical samples $(t(43)=-3.21, p<0.01)$. Similarly, women in the present sample scored lower than women in the clinical samples $(t(357)=-16.12, p<0.001)$ and women in the non-clinical samples $(t(357)=-6.64, p<0.001)$.

\footnotetext{
${ }^{1}$ Normality data of the individual items of the AAQ showed that items $1,3,5,6,7$, and 8 were slightly positively skewed (Skewness/ $\mathrm{SE}>2.0$ ). To rule out that non-normality influenced outcomes of the CFA, a square-root transformation was applied to all item scores. Fit indices of the unitary model using the transformed data were similar to those obtained with the non-transformed data: $\chi^{2} / d f=2.68, \mathrm{CFI}=$ $0.93, \mathrm{NFI}=0.90, \mathrm{TLI} / \mathrm{NNFI}=0.90$, and $\mathrm{RMSEA}=0.07$.
}

Reliability The internal consistency (Cronbach's $\alpha$ ) of the AAQ was 0.74. The test-retest correlation (Pearson's $r$ ), assessed with the subgroup of 30 participants who filled in the AAQ twice, was $0.82 \quad(p<0.001)$. The test-retest correlations of the 9 items (Spearman's $\rho$ ) are shown in Table 1. These were moderate, except the ones for items 4 and 6 that were low.

Concurrent Validity All correlations pertaining to the validity of the AAQ are shown in Table 2. Because the AAQ score was slightly positively skewed, Spearman $\rho$ correlations were calculated to examine associations with the SCL depression scale and the ICG-r. As can be seen in Table 2, correlations between the AAQ and both these measures were positive and significant, attesting to the concurrent validity of the AAQ.

\section{Study 2}

Method

Participants and Procedure Ninety-seven undergraduate students (67 women and 30 men) of Utrecht University participated in this study in return for course credits. Their mean age was 22.1 years $(\mathrm{SD}=2.1)$. After providing informed consent, participants completed questionnaires in groups of two to eight students, in the presence of a research assistant who gave instructions and answered questions that arose.

Measures In addition to the 9-item AAQ (Hayes et al. 2004), participants completed the questionnaires presented below.

The 16-item Depression scale from the Symptom Checklist (SCL-90; Derogatis 1983; Dutch version Arrindell and Ettema 1986) was used to assess depressive symptoms. Cronbach's $\alpha$ in the present sample was 0.91 .

The state version of the State-Trait Anxiety Inventory (STAI-S; Spielberger 1983, Dutch version by Van der Ploeg 1999) was used to assess anxiety symptoms. Respondents rate the degree to which several (positively and negatively keyed) anxiety symptoms are currently present on 4-point scales ranging from 1 ("not at all") to 4 ("very much"). Items are summed to yield a state anxiety score. In this sample, the $\alpha$ amounted to 0.94 .

Domains of the five-factor model were measured with the Neo Five-Factor Inventory (NEO FFI; Costa and McCrae 1992, Dutch version by Hoekstra et al. 2003). The Neo FFI contains five 12-item subscales representing the five personality dimensions of Neuroticism, Extraversion, Agreeableness, Openness to experience, and Conscientiousness. Items are rated on 5-point scale ranging from 1 (strongly disagree) 
Table 2 Associations of the 9-item acceptance and action questionnaire with measures of psychopathology, personality, coping style, and daily hassles

Underlined correlations are Spearman $\rho$ correlations, all other correlations are Pearson $r$ correlations.

$E P C L$ Everyday Problems Checklist, $E P Q$ Eysenck

Personality Questionnaire, ICG-r Inventory of Complicated Grief revised, NEO FFI NEO Five Factor Inventory, SCL Symptom Checklist, STAI State-Trait Anxiety Inventory, WBSI White Bear Suppression Inventory ${ }^{*} p<0.10 ; * * p<0.05$; $* * * p<0.01 ; * * * * p<0.001$

\begin{tabular}{|c|c|c|c|}
\hline & $\begin{array}{l}\text { Study } 1 \\
N=402\end{array}$ & $\begin{array}{l}\text { Study } 2 \\
N=99\end{array}$ & $\begin{array}{l}\text { Study } 3 \\
N=60\end{array}$ \\
\hline \multicolumn{4}{|l|}{ Psychopathology } \\
\hline Complicated grief (ICG-r) & $0.63 * * * *$ & & \\
\hline Depression (SCL) & $\overline{0.63}^{*} * * *$ & $0.56^{* * * *}$ & $0.66 * * * *$ \\
\hline Anxiety (SCL) & & & $0.57 * * * *$ \\
\hline Anxiety (STAI) & & $0.59 * * * *$ & \\
\hline \multicolumn{4}{|l|}{ Personality } \\
\hline Neuroticism (NEO FFI) & & $0.66^{* * * *}$ & \\
\hline Extraversion (NEO FFI) & & $-0.36 * * * *$ & \\
\hline Openness (NEO FFI) & & -0.01 & \\
\hline Agreeableness (NEO FFI) & & $-0.45 * * * *$ & \\
\hline Conscientiousness (NEO FFI) & & $-0.39 * * * *$ & \\
\hline Neuroticism (EPQ) & & & $0.68 * * * *$ \\
\hline \multicolumn{4}{|l|}{ Coping } \\
\hline Thought suppression (WBSI) & & & $0.67 * * * *$ \\
\hline Active coping (COPE) & & $-0.32 * * *$ & -0.20 \\
\hline Positive reinterpretation \& growth (COPE) & & -0.10 & $-0.24 *$ \\
\hline Acceptance (COPE) & & $-0.21 * *$ & $-0.43 * * *$ \\
\hline Venting of emotions (COPE) & & $0 . \overline{17^{*}}$ & $0.24 *$ \\
\hline \multicolumn{4}{|l|}{ Daily hassles } \\
\hline Frequency of daily hassles (EPCL) & & $0.30 * * *$ & \\
\hline Perceived intensity of daily hassles (EPCL) & & $\overline{0.46} * * * *$ & \\
\hline
\end{tabular}

to 5 (strongly agree). The internal consistencies for the five subscales in this sample were: Neuroticism, $\alpha=0.89$, Extraversion, $\alpha=0.80$, Openness, $\alpha=0.67$, Agreeableness, $\alpha=0.61$, Conscientiousness, $\alpha=0.81$.

The Everyday Problems Checklist (EPCL; Vingerhoets and van Tilburg 1994) was used to assess the frequency and the perceived stressfulness of daily hassles. From a list of 114 diverse daily hassles respondents mark the events that they experienced in the previous 2 months. The total number of hassles represents the hassles frequency score. Next, for each of the marked items, respondents rate how strongly they perceive these as upsetting on 4-points scales (ranging from "not at all" to "very much"). The mean score per item represents the perceived stressfulness score. The EPCL has demonstrated good reliability and validity (Vingerhoets and van Tilburg 1994). In the current study, the frequency scale yielded an $\alpha$ of 0.94 .

Coping strategies were assessed with the COPE inventory (Carver et al. 1989), the Dutch version of which was validated by Kleijn et al. (2000). This instrument assesses fifteen different coping strategies, each tapped by four items. Participants rate how frequently they typically use the depicted strategies, on 4-point scales ranging from 1 ("usually do not do this at all") to 4 ("usually do this a lot"). Four scales were administered: Active Coping $(\alpha=0.79)$, Positive Reinterpretation and Growth $(\alpha=0.66)$, and Acceptance $(\alpha=$ 0.62 ) representing adaptive coping strategies, and Venting of Emotions $(\alpha=0.88)$ representing a maladaptive strategy.
Results

Descriptives The AAQ total score was normally distributed (Skewness $=0.17, \mathrm{SE}=0.25$; Kurtosis $=0.37, \mathrm{SE}=0.49$ ). The mean AAQ score of participants was $31.5 \quad(\mathrm{SD}=5.8)$. Scores of men $(M=32.5, \mathrm{SD}=6.5)$ did not differ significantly from those of women $(M=31.1, \mathrm{SD}=5.5 ; t(94)=$ 1.04, $p=0.30)$. As in Study 1, the mean AAQ scores obtained in the present sample were compared with the weighted mean AAQ scores of men and women from the clinical and non-clinical samples in the studies conducted by Hayes et al. (2004). Scores of men in the current sample did not differ from either those observed for men in the clinical samples $(t(29)=-1.81, p=0.08)$ or those observed in the non-clinical samples $(t(43)=-0.62$, $p=0.54)$. However, women in the present sample scored significantly lower than both women in the clinical samples $(t(65)=-9.16, p<0.001)$ and women in the nonclinical samples $(t(65)=-3.34, p<0.01)$ from the research of Hayes et al. (2004).

Internal Consistency The internal consistency of the AAQ was 0.53 .

Concurrent Validity Table 2 shows correlations pertaining to the validity of the AAQ. Although AAQ scores were normally distributed, some of the dependent variables were slightly positively skewed (Skewness/SE $>2.0$ ). For these 
variables, associations with the AAQ were examined using Spearman $\rho$ correlations.

As can be seen in Table 2, the observed correlations with depression and anxiety were positive and highly significant, attesting to the concurrent validity of the AAQ.

Construct Validity Table 2 shows that the AAQ correlated significantly with the frequency index $(\rho=0.30, p<0.01)$ and with the intensity index $(\rho=0.46, p<0.001)$ of the daily hassles scale (EPCL). Differences between correlations were compared using procedures outlined by Meng et al. (1992). Although the former correlation was weaker than the latter, this difference did not reach significance $(Z=1.31, p=0.19)$.

With respect to the association with the five-factor model (NEO FFI), findings were partially in line with the predictions. Specifically, the AAQ was positively associated with neuroticism and inversely linked with extraversion, agreeableness, and conscientiousness. Yet, the AAQ was unrelated to openness.

Finally, with regard to linkages between the AAQ and coping styles (COPE inventory), it was found that the AAQ was significantly and inversely associated with Active Coping and Acceptance, but unrelated to Venting of Emotions and Positive Reinterpretation and Growth (see Table 2).

\section{Study 3}

Method

Participants and Procedure Data were available from 60 outpatients recruited from three outpatient psychotherapy practices. In total, 71 consecutive patients were invited to participate in this study, 60 of which (85\%) agreed to do so. Participants completed questionnaires during the first five contact hours with their therapists. The mean age of the participants was $39.9(\mathrm{SD}=11.5)$ years. Most $(68 \%)$ were women, all were Caucasian, and $68 \%$ of the participants were employed. On average, participants had had 16.5 (SD= 3.1) years of education. Based on semi-structured intakeinterviews, the primary diagnosis was a depressive disorder in 14 patients $(23.3 \%)$, an obsessive compulsive disorder in nine patients $(15 \%)$, social phobia in six cases $(10 \%)$, work-related stress problems in eight cases (13.3\%), relational problems in three cases $(5 \%)$, an adjustment disorder in nine patients $(15.0 \%)$, and another disorder or emotional problem in 11 patients $(18.3 \%)$. Informed consent was obtained from all participants.

Measures Participants completed the 9-item AAQ (Hayes et al. 2004) and the following questionnaires.

Depression and anxiety symptoms were assessed with the 16-item depression scale and the 10-item anxiety scale from the Symptom Checklist (SCL-90; Derogatis 1983, Dutch version by Arrindell and Ettema 1986). In the current sample, these scales yielded $\alpha$ 's of 0.93 and 0.91 respectively.

Thought suppression was measured with the Dutch version of the White Bear Suppression Inventory (WBSI), a 15-item questionnaire tapping the tendency to suppress unwanted thoughts. Respondents rate their agreement with statements representing this tendency on scales ranging from 1 (strongly disagree) to 5 (strongly agree). Items are summed to yield an overall thought suppression score. The English (Wegner and Zanakos 1994) and Dutch versions (Muris et al. 1996) have demonstrated adequate psychometric properties. Cronbach's $\alpha$ in the present sample was 0.92 .

Neuroticism was assessed with the 12-item N-scale from the shortened Eysenck Personality Questionnaire (EPQ; Eysenck et al. 1985), the Dutch version of which was developed and validated by Sanderman et al. 1995). Respondent indicate their agreement with each item, using a forced-choice response format (yes vs. no). The $\alpha$ was 0.83 .

Coping strategies were assessed with the COPE inventory (Carver et al. 1989; Dutch version by Kleijn et al. 2000). As in Study 2, only the scales Active Coping (in this sample $\alpha=0.70)$, Positive Reinterpretation and Growth $(\alpha=$ $0.79)$, Acceptance $(\alpha=0.71)$, and Venting of Emotions $(\alpha=$ $0.81)$ were administered.

\section{Results}

Descriptives The skewness (0.21, $\mathrm{SE}=0.31)$ and kurtosis $(0.77, \mathrm{SE}=0.61)$ of the AAQ total score indicated that this score was normally distributed. The mean score of participants on the AAQ was $36.0(\mathrm{SD}=7.4)$. Scores of men $(M=36.5, \mathrm{SD}=7.1)$ did not differ significantly from those of women $(M=35.8, \mathrm{SD}=7.6 ; t(58)=0.34, p=0.74)$. AAQ scores were also compared across diagnostic subgroups. To increase power, diagnostic subgroups were combined into three categories, namely mood disorders $(n=14)$, anxiety disorders $(n=17)$, and other disorders and problems $(n=29)$. No significant between-group differences were observed $(F(2,57)=0.68, p=0.64)$. In a similar vein, scores on all dependent measures that were used showed no differences as a function of diagnostic status $\left(F{ }^{\prime} s<2.78\right.$, p's $>0.07)$.

Similar to Studies 1 and 2, mean AAQ scores of men and women in the present sample were compared with the weighted mean AAQ scores of men and women from the clinical samples and non-clinical samples included in the study of Hayes et al. (2004). Scores of men in the current sample did not differ from those of men in either the clinical samples $(t(18)=1.16, p=0.26)$ or the non-clinical samples $(t(18)=2.02, p=0.06)$. Similarly, scores of women did not differ from the scores of women in the clinical 
samples $(t(40)=-1.31, p=0.20)$ and non-clinical samples $(t(40)=2.0, p=0.053)$.

Internal Consistency The internal consistency of the AAQ was 0.65 .

Concurrent Validity Correlations of the AAQ with other measures are shown in Table 2. Both the AAQ scores and dependent variables were normally distributed. Hence, Pearson $r$ correlations were calculated in all instances. In support of the concurrent validity of the AAQ, correlations with symptoms of depression and anxiety were positive and significant (Table 2).

Construct Validity As expected, the AAQ was significantly associated with both neuroticism and thought suppression (see Table 2). ${ }^{2}$ To examine if the AAQ remained associated with anxiety and depression after taking the shared variance with neuroticism and thought suppression into account, partial correlations were calculated between the AAQ and depression and anxiety. The AAQ remained significantly associated with depression after controlling for neuroticism $\left(r_{\mathrm{p}}=0.33, p<0.05\right)$, and also after controlling for thought suppression $\left(r_{\mathrm{p}}=0.45, p<0.001\right)$. The AAQ also remained significantly associated with anxiety after controlling for neuroticism $\left(r_{\mathrm{p}}=0.26\right)$ and also after controlling for thought suppression $\left(r_{\mathrm{p}}=0.28, p\right.$ 's $\left.<0.05\right)$. Taken together, these findings show that the AAQ predicts depression and anxiety above and beyond neuroticism and thought suppression, thereby attesting to the incremental validity of the AAQ.

Finally, Table 2 shows that the AAQ was significantly inversely related with the coping strategy Acceptance. Conversely, the linkages with Positive Reinterpretations and Growth $(r=-0.24, p=0.06)$, and Venting of Emotions $(r=0.24, p=0.07)$ only approached significance. The AAQ was not related to Active Coping.

Comparison of AAQ Scores Across Study Samples The AAQ total scores were compared across the three samples included in the current research. An analysis of variance revealed that scores differed across the groups $(F(2,556)=$ 14.1, $p<0.001)$. Post-hoc tests showed that the clinical sample scored significantly higher than both the bereaved sample and the student sample $(p$ 's $<0.001)$, whereas these two latter samples did not differ from each other $(p=0.49)$.

\footnotetext{
${ }^{2}$ The correlation between the AAQ and the WBSI was similar in magnitude to the correlation of 0.50 that was observed in a sample of 50 female borderline personality disorder patients $(Z=1.34, p=0.18)$ and higher than the correlation of 0.44 found among 202 university students $(Z=2.25, p<0.05)$; both described in the research of Hayes et al. (2004).
}

Additional Analyses with an 8 Item Version of the $A A Q$ Study 1 showed that item 6 was a relatively weak performing item, as evidenced by both a non-significant factor loading on the underlying experiential avoidance factor, as well as a low test-retest reliability. Although the key goal of this study was to evaluate the AAQ as it was originally developed (the 9-item format), it was considered relevant to examine the potentially detrimental effect of this poor performing item on the reliability and validity of the AAQ. Specifically, all analyses were rerun using an adjusted 8 item version (i.e., deleting item 6). This 8 item version fitted the data slightly better than did the 9 item version $\left(\chi^{2} / d f=2.65, \mathrm{CFI}=0.95, \mathrm{NFI}=0.93, \mathrm{TLI} / \mathrm{NNFI}=\right.$ 0.93 , and RMSEA $=0.06$ ), and also showed slightly higher internal consistency values in all three samples $(\alpha=0.74$, $\alpha=0.59$, and $\alpha=0.66$, respectively). However, no meaningful changes emerged with respect to the validity. Correlations of the 8 item version with measures of psychological functioning were almost identical to those of the 9 item version, and no differences in significance levels of the observed correlations emerged.

\section{Discussion}

The current studies examined the psychometric properties of the Dutch version of the 9-item AAQ, originally developed by Hayes et al. (2004) to assess experiential avoidance as conceptualized within ACT (Hayes et al. 1999). Three studies were conducted with a total number of 559 participants, including a large group of bereaved individuals, a non-clinical sample of undergraduate students, and a clinical sample of adult outpatients.

In Study 1, CFA was used to examine the fit of a model in which the items of the AAQ constituted a unitary factor of experiential avoidance. Findings showed that, after a minor adjustment (i.e. allowing error-terms of two comparable items to be correlated) this model fitted the data well. Findings are consistent with those of Hayes et al. (2004) and provide additional evidence that a common core process cuts through different aspects of experiential avoidance (e.g., negative evaluation of private events, inability to take action when faced with such events, high need for emotional and cognitive control). Yet, it is noteworthy that the item phrased "When I evaluate something negatively, I usually recognize that this is just a reaction, not an objective fact" did not load significantly on the experiential avoidance factor. Although this finding suggests that this item may not be a good indicator of experiential avoidance, it appears that the finding is specific to the sample examined (i.e., bereaved adults). In fact, in 
the study of Hayes et al. (2004) this item showed a factorloading of 0.56 . At this point, it is difficult to offer a plausible explanation for this discrepancy. If future studies replicate the poor performance of this item, it should perhaps be removed from the AAQ.

The test-retest correlation found in Study 1 indicated that the total score of the AAQ was relatively stable across an average period of three weeks. This finding links up with earlier studies that have shown that the AAQ total score is reasonably stable across periods of 5 to 6 weeks $(r=0.71$; Barraca Mairal 2004) and 4 months $(r=0.64$; Hayes et al. 2004). However, the stability of the individual items was less satisfactory, with items 4 and 6 showing poor stability. Hence, it appears that experiential avoidance as a broad tendency is relatively stable, whereas specific manifestations of this inclination may vary considerably across time and across situations. This fits the notion that the multiple features of experiential avoidance are situated actions rather than stable traits (Hayes et al. 2004).

The internal consistency of the AAQ was adequate in Study 1, but quite low in the other two samples examined (Study 2 in particular), and also lower relative to the original instrument $(\alpha=0.70$; Hayes et al. 2004) and the Spanish version $(\alpha=0.74$; Baracca Mairal 2004). Although the cause of this somewhat disappointing result among undergraduates is unclear, this finding is relevant for the discussion about the appropriateness of applying conventional psychometric criteria to the evaluation of measures of experiential avoidance. Specifically, as is noted by Hayes et al. (2004), from the viewpoint of ACT/RFT, tendencies to engage in experiential avoidance are not seen as representing a stable, internally consistent underlying trait, but, instead, are situated actions that have many different behavioral features. In this context, these authors noted "the alpha level seems likely to remain an issue because the underlying theory $[\ldots]$ suggests that the psychological processes being measured necessarily will include events that can participate in other, more specific processes" (p. 572).

With regard to the validity of the AAQ, in all three studies the AAQ was found to be strongly linked with measures tapping different forms of psychopathology, including symptoms of anxiety, depression, and complicated grief. These findings link up with previous research that supported the concurrent validity of the AAQ (Barraca Mairal 2004; Hayes et al. 2004) and with the growing body of research that points to the importance of experiential avoidance in psychopathology (Hayes et al. 2006). For instance, comparable to the present findings, Hayes et al. (2004) found the AAQ to be significantly associated with depression (with correlations ranging from 0.36 to 0.72 ) and anxiety (with correlations ranging from 0.35 to 0.58$)$.
Study 3 showed that the AAQ was also significantly associated with related constructs of neuroticism and thought suppression. These findings replicate earlier work also showing linkages between experiential avoidance and these variables (e.g., Bond and Bunce 2003; Hayes et al. 2004; Kashdan et al. 2006). Importantly, associations of the AAQ with depression and anxiety remained significant after controlling for the shared variance of the AAQ with these related constructs. These findings attest to the incremental validity of the AAQ, by showing that this measure taps a phenomenon that goes beyond neuroticism and thought suppression alone.

Studies 2 and 3 yielded several other findings supporting the validity of the AAQ. First, the linkage with the perceived stressfulness of daily hassles was stronger (albeit not significantly so) than the linkage with the actual frequency of these stressors. Second, in addition to the expected positive association between the AAQ and neuroticism (cf. Bond and Bunce 2003; Kashdan et al. 2006), individuals scoring higher on the AAQ were lower in extraversion, agreeableness, and conscientiousness. Finally, across studies a significant negative association between the AAQ and the coping strategy "acceptance" was observed.

Several limitations of the current studies deserve mention. First, women were overrepresented in Study 1. Hence, it remains to be determined to what extent findings on the factor structure of the AAQ can be generalized to men. Since the sample consisted of bereaved individuals only, future studies examining the factor structure across different populations appear warranted. Second, it should be noted that the internal consistency of some assessment instruments was modest (e.g., several subscales of the COPE in Study 2). Third, the clinical sample used in Study 3 was relatively small. Limited statistical power may therefore account for some of the non-significant associations with indices of coping style. Another caveat is that this sample was heterogeneous with respect to diagnostic status. Although this might indicate generalizability of findings to broad clinical populations, future studies should examine to what extent the AAQ performs differently across diagnostic subgroups. Finally, ethnicity data were not registered in Studies 1 and 2, whereas all participants in Study 3 were Caucasian. Hence, it was not possible to examine if the differences in AAQ scores between different ethnic groups observed in the study of Hayes et al. (2004) also existed in the current Dutch samples.

Notwithstanding these limitations, the current studies provide important information about the psychometric properties of the Dutch version of the AAQ. Overall, the results are in line with earlier studies that have examined the psychometric properties of the AAQ (Baracca Mairal 
2004; Hayes et al. 2004). In addition, the findings add to the impressive body of work that has provided empirical support for the ACT/RFT model of experiential avoidance (Hayes et al. 2006). Importantly, the similarity between the present findings and studies using the Spanish version (Baracca Mairal 2004) and original English version (Hayes et al. 2004, 2006) suggests that the measure can reliably be applied across different Western cultures. However, further improvement of the 9-item AAQ seems warranted given some of its weaker psychometric properties (e.g., its moderate internal consistency), as well as the rapidly growing knowledge on the nature and importance of experiential avoidance. As noted by Hayes et al. (2004), the 9-item AAQ is meant as a starting point for the development of more comprehensive and/or disorderspecific measures of experiential avoidance. Although the field is indeed likely to benefit from future scale development, the current studies suggest that, in its present form, the 9-item AAQ is a useful tool for the purpose that it was designed for, namely exploring experiential avoidance in large population-based studies.

Open Access This article is distributed under the terms of the Creative Commons Attribution Noncommercial License which permits any noncommercial use, distribution, and reproduction in any medium, provided the original author(s) and source are credited.

\section{References}

Arbuckle, J. L. (2003). Amos 5.0 update to the Amos user's guide. Chicago: Smallwaters Corporation.

Arrindell, W. A., \& Ettema, J. (1986). SCL-90. Een multidimensionele psychopathologie-indicator. [SCL-90: manual for a multidimensional indicator of psychopathology.] Lisse: Swets \& Zeitlinger.

Baracca Mairal, J. (2004). Spanish adaptation of the Acceptance and Action Questionnaire. International Journal of Psychology and Psychological Therapy, 4, 505-515.

Barlow, D. H. (2002). Anxiety and its disorders: The nature and treatment of anxiety and panic (2nd ed.). New York: Guilford.

Blackledge, J. T., \& Hayes, S. C. (2001). Emotion regulation in acceptance and commitment therapy. Journal of Clinical Psychology, 57, 243255.

Boelen, P. A., van den Bout, J., de Keijser, J., \& Hoijtink, H. (2003). Reliability and validity of the Dutch version of the Inventory of Traumatic Grief. Death Studies, 27, 227-249.

Bond, F. W., \& Bunce, D. (2003). The role of acceptance and job control in mental health, job satisfaction, and work performance. Journal of Applied Psychology, 88, 1057-1067.

Carver, C. S., Scheier, M. F., \& Weintraub, J. K. (1989). Assessing coping strategies: A theoretically based approach. Journal of Personality and Social Psychology, 56, 267-283.

Costa, Jr., P. T., \& McCrae, R. R. (1992). Revised NEO Personality Inventory (NEO-PI-R) and NEO Five-Factor Inventory (NEO-FFI) professional manual. Odessa, FL: Psychological Assessment Resources.
Derogatis, L. R. (1983). SCL-90-R: Administration, scoring, and procedures. Manual II (2nd ed.). Towson, Maryland: Clinical Psychometric Research.

Durrett, C., \& Trull, T. J. (2005). An evaluation of evaluative personality terms: A comparison of the big seven and five-factor model in predicting psychopathology. Psychological Assessment, 17, 359-368.

Eysenck, S. B. G., Eysenck, H. J., \& Barrett, P. (1985). A revised version of the psychoticism scale. Personality and Individual Differences, 6, 21-29.

Gross, J. J., \& Levenson, R. W. (1997). Hiding feelings: The acute effects of inhibiting negative and positive emotion. Journal of Abnormal Psychology, 106, 95-103.

Hayes, S. C. (1994). Content, context, and the type of psychological acceptance. In S. C. Hayes, N. S. Jacobson, V. M. Follette, \& M. J. Dougher (Eds.) Acceptance and change: Content and context in psychotherapy (pp. 13-32). Reno NV: Context.

Hayes, S. C., Barnes-Holmes, D., \& Roche, B. (Eds.) (2001). Relational frame theory: A post-Skinnerian account of human language and cognition. New York: Plenum.

Hayes, S. C., Luoma, J., Bond, F., Masuda, A., \& Lillis, J. (2006). Acceptance and commitment therapy: Model, processes, and outcomes. Behaviour Research and Therapy, 44, 1-25.

Hayes, S. C., Strosahl, K., \& Wilson, K. G. (1999). Acceptance and commitment therapy: An experiential approach to behavior change. New York: Guilford.

Hayes, S. C., Strosahl, K., Wilson, K. G., Bissett, R. T., Pistorello, J., Toarmino, D., et al. (2004). Measuring experiential avoidance: A preliminary test of a working model. The Psychological Record, 54, 553-578.

Hayes, S. C., Wilson, K. G., Gifford, E. V., Follette, V. M., \& Strohsahl, K. (1996). Experiential avoidance and behavioral disorders: A functional dimensional approach to diagnosis and treatment. Journal of Consulting and Clinical Psychology, 64, 1152-1168.

Hoekstra, H. A., de Fruyt, F., \& Ormel, J. (2003). Neo-Persoonlijkheidsvragenlijsten: NEO-PI-R, NEO-FFI [Neo personality questionnaires: NEO-PI-R, NEO-FFI]. Lisse: Swets Test Services.

Kashdan, T. B., Barrios, V., Forsyth, J. P., \& Steger, M. F. (2006). Experiential avoidance as a generalized psychological vulnerability: Comparisons with coping and emotion regulation strategies. Behaviour Research and Therapy, 44, 1301-1320.

Kleijn, W. Chr, van Heck, G. L., \& van Waning, A. (2000). Ervaringen met een Nederlandse bewerking van de COPE copingvragenlijst: De COPE-Easy [Experiences with a Dutch adaptation of the COPE coping questionnaire: The COPE-Easy]. Gedrag \& Gezondheid, 28, 213-226.

Linehan, M. M. (1993). Cognitive-behavioral treatment of borderline personality disorder. New York: Guilford.

McCrae, R. R., \& Costa, P. T., Jr. (1999). A five-factor theory of personality. In L. A. Pervin \& O. P. John (Eds.), Handbook of personality: Theory and research (2nd ed., pp. 139-153). New York: Guilford Press.

Meng, X. L., Rosenthal, R., \& Rubin, D. B. (1992). Comparing correlated correlation coefficients. Psychological Bulletin, 111, $172-175$.

Muris, P., Merckelbach, H., \& Horselenberg, R. (1996). Individual differences in thought suppression. The White Bear Suppression Inventory: Factor structure, reliability, validity and correlates. Behaviour Research and Therapy, 34, 501-513.

Prigerson, H. G., \& Jacobs, S. C. (2001). Traumatic grief as a distinct disorder: A rationale, consensus criteria, and a preliminary empirical test. In M. S. Stroebe, R. O. Hansson, W. Stroebe, \& H. A. W. Schut (Eds.) Handbook of bereavement research: Consequences, coping, and care (pp. 613-647). Washington DC: American Psychological Association. 
Sanderman, R., Arrindell, W. A., Ranchor, A., Eysenck, H. J., \& Eysenck, S. B. G. (1995). Het meten van persoonlijkheidskenmerken met de Eysenck Personality Questionnaire: een handleiding [Measuring personality characteristics with the Eysenck Personality Questionnaire: A manual]. Groningen: Noordelijk Centrum voor Gezondheidsvraagstukken.

Segal, Z. V., Williams, J. M. G., \& Teasdale, J. T. (2001). Mindfulness-based cognitive therapy for depression: A new approach to preventing relapse. New York: Guilford.

Speilberger, C. D. (1983). Manual for the State-Trait Anxiety Inventory (STAI). PaloAlto, CA: Consulting Psychologists Press.
Trull, T. J., \& Sher, K. J. (1994). Relationship between the Five Factor Model of personality and axis I disorders in a non-clinical sample. Journal of Abnormal Psychology, 2(103), 350-360.

Van der Ploeg, H. M. (1999). Handleiding bij de Zelf-Beoordelings Vragenlijst, $Z B V$ [Manual for the Stait-Trait Axiety Inventory]. Lisse: Swets \& Zeitlinger.

Vingerhoets, A. J. J. M., \& van Tilburg, M. A. L. (1994). De alledaagse problemenlijst [The Dutch Daily Hassles Scale]. Lisse: Swets \& Zeitlinger.

Wegner, D. M., \& Zanakos, S. (1994). Chronic thought suppression. Journal of Personality, 62, 615-640. 